\title{
GLOBAL OBSERVABILITY AND DETECTABILITY ANALYSIS OF UNCERTAIN REACTION SYSTEMS
}

\author{
Jaime A. Moreno ${ }^{*, 1,2}$ Denis Dochain ${ }^{* *, 2}$ \\ * Instituto de Ingeniería, UNAM, Automatización, Cd. \\ Universitaria, C.P.04510 México D.F., MEXICO, \\ JMorenoP@iingen.unam.mx, Fax: ++(52-55) 56228130 \\ ** CESAME, Université Catholique de Louvain, 4-6 avenue \\ G. Lemaitre, 1348 Louvain-la-Neuve, Belgium. \\ dochain@csam.ucl.ac.be
}

\begin{abstract}
Observation issues for reaction systems is of fundamental importance since there is a lack of sensors and the uncertainties can be very high. The objective of this work is to propose a methodology to make a global analysis of observability and detectability of such systems, with a particular concern about the design of unknown input observers. Copyright (C)2005 IFAC
\end{abstract}

Keywords: Observability, indistinguishability, detectability, uncertain reaction systems, Unknown input observer

\section{INTRODUCTION}

Reaction systems is a class of nonlinear dynamical systems that is widely used in areas such as chemical and biochemical engineering, biomedical engineering, biotechnology, ecology, etc. (Robust) Observation issues for this class of systems is of fundamental importance since there is a lack of sensors and the uncertainties can be very high. It is not surprising that there is an intensive research activity to design observers, or software sensors, for these systems (Bastin and Dochain, 1990; Dochain and Vanrolleghem, 2001; Dochain, 2003). It is well known, that the existence of observers for dynamical systems is intrinsically related to the observability, or detectability, of the system. How-

\footnotetext{
1 Work done during a stay at the ISR, University of Stuttgart, Germany. Thanks to DGAPA-UNAM, project PAPIIT IN111905-2 for financial support.

2 This paper includes results of the EOLI project that is supported by the INCO program of the European Community, Contract number ICA4-CT-2002-10012. The scientific responsibility rests with the authors.
}

ever, there are few studies in the literature dealing with the observability, detectability properties of reacting systems. Most of these studies are local in nature (Dochain and Chen, 1992). However, a satisfactory solution to the observation problem is global, since it seems unreasonably to require that the initial condition of the system is up to a small error known, when one of the motivations to use an observer is precisely the lack of information on this initial state!

The objective of this work is to propose a methodology to make a global analysis of observability and detectability of reaction systems. This is in contrast to the usual local results of the literature. When there are no uncertainties the usual method to do a global observability analysis is by means of the observability map (Zeitz, 1989; Gauthier and Kupka, 2001). However, there is no systematic method to study detectability for nonlinear systems. In this work a methodology is presented to carry out a global observability/detectability analysis of uncertain reaction systems, when the 
uncertainty is represented as an arbitrary and unknown input signal. For this kind of systems no method is known to study observability. This method is an extension for the uncertain case of the idea used for the analysis of the induction machine under sensorless operation (Ibarra-Rojas et al., 2004). Other approaches are given in (Ponzoni et al., 2004), based on structural information, but restricted to non dynamical representations of the plant in steady state. The cornerstone of this result is the strongly indistinguishable trajectories concept (internal trajectories of a system that are different under the same input/output behavior under uncertain inputs) and the main result is the characterization of the complete set of this kind of functions for the uncertain reaction system, including both types of indistinguishable trajectories: the divergent (non-observable) ones, i.e. those which are not possible to identify by input/output measurements, and the asymptotically convergent (detectable) ones, i.e. those that can be determined by the input/output behavior asymptotically. The proposed methodology achieves the desired characterization by constructing a nonlinear dynamical system, called strongly indistinguishable dynamics, whose set of solution trajectories corresponds to the aforementioned set of strongly indistinguishable behaviors, i.e. every solution trajectory of this system is an strongly indistinguishable trajectory for the studied system. Some sufficient conditions for an uncertain reaction system to be observable/detectable or to be not observable/not detectable will be given.

The paper is organized as follows. A general model of reaction systems is given in Section 2 . Global observability/detectability properties are introduced for uncertain systems and their relationship with the existence of observers is studied in Section 3. In Sections 4 and 5 the observability properties of uncertain reaction systems is analyzed and their consequences for observer design is given in Section 6 .

\section{MODEL OF REACTION SYSTEMS}

A general state-space model of reaction systems is generally obtained from mass and energy balances (Bastin and Dochain, 1990; Dochain et al., 1992; Dochain and Vanrolleghem, 2001) :

$$
\begin{aligned}
\frac{d c}{d t} & =\bar{K} \varphi(c, T)+D\left(c_{i n}-c\right)-Q_{c}(c, T)+\bar{F} \\
\frac{d T}{d t} & =-\frac{1}{\rho C_{p}} \Delta H^{T} \varphi(c, T)+D\left(T_{i n}-T\right)-Q_{T}
\end{aligned}
$$

where $c \in \mathbb{R}^{n-1}, T \in \mathbb{R}, \bar{K} \in \mathbb{R}^{(n-1) \times q}, \varphi \in \mathbb{R}^{q}$, $D \in \mathbb{R}, Q_{c} \in \mathbb{R}^{n-1}, \bar{F} \in \mathbb{R}^{n-1}, \rho \in \mathbb{R}, C_{p} \in \mathbb{R}$, $\Delta H \in \mathbb{R}^{q}, Q_{T} \in \mathbb{R}, c_{\text {in }} \in \mathbb{R}^{n-1}$ and $T_{\text {in }} \in \mathbb{R}$ are the component concentration vector, the temperature, the stoichiometric coefficient matrix, the reaction rate vector, the dilution rate, the gaseous outflow rate vector, the feedrate vector, the density, the heat capacity, the reaction heat vector, the heat exchange term, and the inlet concentration vector and temperature, respectively. In most applications the measured variables are a subset of the state variables or, more generally, a linear combination of them.

This system can be written in a compact and generalized form as

$$
\Sigma_{R}:\left\{\begin{array}{l}
\dot{x}=K \varphi(x)+D\left(x_{i n}-x\right)-Q(x)+F \\
y=C x
\end{array}\right.
$$

where $x \in \mathbb{R}^{n}$ is the state, and $y \in \mathbb{R}^{m}$ is the output vector. This model includes also the case when several reactors are considered, for which $D \in \mathbb{R}^{n \times n}$ is a matrix. In practice the knowledge of the model is usually very uncertain, since the parameters and nonlinearities of the system are difficult to identify, and they change over the time. In particular, the reaction rates are usually poorly known. This makes the observation problem challenging. In this paper we shall concentrate on the case when the reaction rates are unknown, but the rest of the model is assumed to be known, i.e. $u=F-Q(x)+D x_{i n}$ is considered a known signal (Bastin and Dochain, 1990). This uncertainty will be modeled as an (arbitrary) unknown input $w=\varphi(x)$.

\section{OBSERVABILITY AND DETECTABILITY CONCEPTS FOR NONLINEAR UNCERTAIN SYSTEMS AND EXISTENCE OF OBSERVERS}

Since the uncertainties in the reaction system (1) will be represented by unknown inputs, let us consider a general nonlinear system described by

$$
\Sigma:\left\{\begin{array}{l}
\dot{x}=f(x, u, w), \quad x(0)=x_{0} \\
y=h(x),
\end{array}\right.
$$

where $x \in \mathbb{R}^{n}$ is the state vector, $u \in \mathbb{R}^{p}$ is the input vector, $w \in \mathbb{R}^{q}$ is a vector of unknown inputs, $y \in \mathbb{R}^{m}$ is the output vector, and $f$ and $h$ are sufficiently smooth functions. The solution of (2) passing through $x_{0}$ at $t=0$ and corresponding to the input function $u(\cdot)$ and $w(\cdot)$ is denoted as $x\left(t, x_{0}, u(\cdot), w(\cdot)\right)$. In a similar way, let us denote the output $y\left(t, x_{0}, u(\cdot), w(\cdot)\right)=$ $h\left(x\left(t, x_{0}, u(\cdot), w(\cdot)\right)\right)$. Whenever there is no confusion, these will be simply denoted as $x(t)$ and $y(t)$. Let us assume in addition that the system $\Sigma$ is complete, i.e. the state trajectories $x(t)$ are defined for every $t \geq 0$, every initial condition $x_{0} \in \mathbb{R}^{n}$ and every input $u(\cdot) \in \mathcal{U}$, and $w(\cdot) \in \mathcal{W}$, where $\mathcal{U}, \mathcal{W}$ are classes of input functions.

For systems without unknown inputs a basic concept is that of indistinguishable states (Hermann 
and Krener, 1977). Roughly speaking two states are said to be indistinguishable if they are different although both the input and the output of the system are identical. The importance of this definition comes from the fact that observer's existence for the system strongly relies on the existence (and the type) of this kind of functions. For systems with unknown inputs (2) similar concepts can be introduced.

Definition 1. (Strong Indistinguishability and Observability and Strong(*) Detectability) Consider for system (2) an input $u(\cdot)$, an initial condition $x \in \mathbb{R}^{n}$ and an unknown input $w(\cdot)$. If $\bar{x} \in \mathbb{R}^{n}, \bar{x} \neq x$, is such that $y(t, x, u(\cdot), 0)=y(t, \bar{x}, u(\cdot), w(\cdot)), \forall t \in[0, \infty)$ and for some $w(\cdot) \in \mathcal{W}$, then $\bar{x}$ is a strongly $u(\cdot)$ indistinguishable state of $x . \mathcal{I}_{(u, x)}^{U I}$ denotes the set of strongly $u(\cdot)$-indistinguishable states of $x$.

System (2) is strongly observable if for every $x \in$ $\mathbb{R}^{n}$ and every $u(\cdot) \in \mathcal{U}, \mathcal{I}_{(u, x)}^{U I}=\{x\}$.

System (2) is strongly detectable if for every $x \in \mathbb{R}^{n}$, every $u(\cdot) \in \mathcal{U}$ and for every $\bar{x} \in$ $\mathcal{I}_{(u, x)}^{U I}$ and the corresponding $w(\cdot)$ that renders $\bar{x}$ indistinguishable $\lim _{t \rightarrow \infty} \| x(t, \bar{x}, u(t), w(\cdot))-$ $x(t, x, u(t), 0) \|=0$.

System (2) is strong ${ }^{*}$ detectable if for some $x, \bar{x} \in$ $\mathbb{R}^{n}, u(\cdot) \in \mathcal{U}$ and $w(\cdot) \in \mathcal{W}$ it happens that $\lim _{t \rightarrow \infty}\|y(t, \bar{x}, u(t), w(t))-y(t, x, u(t), 0)\|=0$, then it follows that $\lim _{t \rightarrow \infty} \| x(t, \bar{x}, u(t), w(t))-$ $x(t, x, u(t), 0) \|=0$.

Note that strong detectability excludes the existence of diverging strongly indistinguishable trajectories. It may be surprising that two detectability definitions have been introduced. For LTI systems without unknown inputs it is well-known that if the unobservable subsystem is asymptotically stable then if as $t \rightarrow \infty y(t) \rightarrow 0$ then $x(t) \rightarrow 0$. However, for continuous time systems with unknown inputs this is not longer the case, as has been pointed out by (Hautus, 1983).

Remark 2. It is clear that strong* detectability implies strong detectability, but the converse is not true. Moreover, strong observability implies strong detectability, but it does not necessarily imply strong* detectability.

These properties are indeed related to the existence of Unknown Input Observers (UIO).

Definition 3. (UI Observer) Consider a system

$$
\Omega:\left\{\begin{array}{l}
\dot{z}=\varphi(z, u, y), \quad z(0)=z_{0} \\
\hat{x}=\chi(z, u, y),
\end{array}\right.
$$

where $z \in \mathbb{R}^{r}$ is the state vector and $\varphi, \chi$ are functions defined in $(z, u, y) \in \mathbb{R}^{r} \times \mathbb{R}^{p} \times$
$\mathbb{R}^{m} \cdot z\left(t, z_{0}, u, y\right)$ denotes a solution of (3) passing through $z_{0}$ at $t=0$ and corresponding to $(u, y)$. System (3) is called an unknown input observer (UIO) for system (2) if $\exists z_{0} \in \mathbb{R}^{r}$ such that $\forall x_{0} \in \mathbb{R}^{n}, \forall u(\cdot) \in \mathcal{U}$ and $\forall w(\cdot) \in \mathcal{W}$

$\lim _{t \rightarrow \infty}\left\|\hat{x}\left(t, z_{0}, u, y\left(t, x_{0}, u, w\right)\right)-x\left(t, x_{0}, u, w\right)\right\|=0$.

Note that in this definition no major restriction has been imposed on the observer, except for the convergence of the observer for every trajectory, i.e. initial condition and input, of the plant.

For LTI systems it is shown by (Hautus, 1983) that strong* detectability is equivalent to the existence of an UIO and that strong detectability or observability are not sufficient for the existence of an UIO. Let us now partially generalize the concept for nonlinear systems.

The following result is indeed valid for every reasonable definition of observer, since it depends on a structural restriction of the system and not on the structure of the observer.

Lemma 4. If system (2) has an unknown input global observer, then it is strong* detectable. Moreover, if the convergence of the observer can be assigned arbitrarily fast, then it is strongly observable.

PROOF. Suppose that system (2) is not strong* detectable. Then there exist an input $u(\cdot)$, two states $x_{1}, x_{2} \in \mathbb{R}^{n}$ and an unknown input $w(\cdot)$ such that as $t \rightarrow \infty, y\left(t, x_{1}, u, 0\right) \rightarrow y\left(t, x_{2}, u, w\right)$, but $x\left(t, x_{1}, u, 0\right)-x\left(t, x_{2}, u, w\right) \nrightarrow 0$. For an UI (global) observer $\Omega$ of $\Sigma$ there exists $z_{0} \in \mathbb{R}^{r}$ such that $\hat{x}\left(t, z_{0}, u, y\left(t, x_{1}, u, 0\right)\right) \rightarrow x\left(t, x_{1}, u, 0\right)$ and $\hat{x}\left(t, z_{0}, u, y\left(t, x_{2}, u, w\right)\right) \rightarrow x\left(t, x_{2}, u, w\right)$. Note $\hat{x}\left(t, z_{0}, u, y\left(t, x_{1}, u, 0\right)\right) \rightarrow \hat{x}\left(t, z_{0}, u, y\left(t, x_{2}, u, w\right)\right)$. But since

$$
\begin{aligned}
& \lim _{t \rightarrow \infty}\left\{x\left(t, x_{1}, u, 0\right)-x\left(t, x_{2}, u, w\right)\right\}= \\
& \quad=\lim _{t \rightarrow \infty}\left\{x\left(t, x_{1}, u, 0\right)-\hat{x}\left(t, z_{0}, u, y_{1}\right)\right\}+ \\
& +\lim _{t \rightarrow \infty}\left\{x\left(t, x_{2}, u, w\right)-\hat{x}\left(t, z_{0}, u, y_{2}\right)\right\}=0,
\end{aligned}
$$

the assumption on $x_{1}, x_{2}$ is contradicted.

To prove the second assertion, suppose by contradiction, that there are two convergent indistinguishable trajectories. Then an observer cannot converge to the correct state at a rate greater than the convergence rate of the trajectories.

From this lemma it is clear that for studying the possibility of estimating state trajectories of a given dynamical system, one can first investigate the existence of robustly indistinguishable trajectories and then determine if they are converging or not, i.e. if the system is robustly detectable or observable. 


\section{THE ERROR AND INDISTINGUISHABLE DYNAMICS OF REACTION SYSTEMS}

The conditions given above are abstract and no checkable conditions were given. The objective of this section is to introduce a dynamical interpretation of the concepts introduced in the previous section. This interpretation will be used in the following section to derive necessary conditions for the existence of global observers. This dynamic characterization, although possible for a general nonlinear system, will be derived here for the case of interest in the paper, the uncertain reaction system (1). It will be assumed that the model is well-known, except for the reaction rates that are completely unknown, so that they can be considered as unknown inputs.

The following Gedanken-experiment leads to the desired characterization of strong* and strong detectability: Two identical systems, but with different initial conditions and unknown inputs, evolve in time

$$
\begin{aligned}
& \dot{x}_{i}=K w_{i}-D x_{i}+u, x_{i}(0)=x_{i 0}, \\
& y_{i}=C x_{i}, \quad i=1,2 .
\end{aligned}
$$

Introducing the variables $x=x_{1}, y=y_{1}, w=w_{1}$ and $\tilde{x}=x_{1}-x_{2}, \tilde{y}=y_{1}-y_{2}, \tilde{w}=w_{1}-w_{2}$, the error dynamics of the plant is given as

$$
\begin{aligned}
& \dot{x}=K w-D x+u, x(0)=x_{0}, \\
& \dot{\tilde{x}}=K \tilde{w}-D \tilde{x}, \tilde{x}(0)=\tilde{x}_{0}, \\
& y=C x, \\
& \tilde{y}=C \tilde{x} .
\end{aligned}
$$

Since for this system the evolution of $x$ does not affect the error state $\tilde{x}$, the basic properties can be studied via the reduced linear system

$$
\begin{aligned}
& \dot{\tilde{x}}=-D \tilde{x}+K \tilde{w}, \tilde{x}(0)=\tilde{x}_{0}, \\
& \tilde{y}=C \tilde{x} .
\end{aligned}
$$

Consider also the linear Differential-Algebraic (DA) system,

$$
\begin{aligned}
& \tilde{x}=-D \tilde{x}+K \tilde{w}, \tilde{x}(0)=\tilde{x}_{0}, \\
& 0=C \tilde{x} .
\end{aligned}
$$

derived from (5) by setting $\tilde{y}(t)=0$ for all $t \geq 0$. This system will be called the (reduced) Strongly Indistinguishable Dynamics of the plant (1) with unknown reaction rates. Strong $(*)$ detectability and observability of the plant can be determined and characterized analyzing the properties of the solution set of the Strong Indistinguishable Dynamics. The following result is a simple consequence of the definitions.

Lemma 5. Consider the system (1), with unknown reaction rates.

(1) Two trajectories are strongly indistinguishable if and only if they are of the form $x\left(t, x_{0}, u(t), w(\cdot)\right)$ and $x\left(t, x_{0}, u(t), w(\cdot)\right)+$ $\tilde{x}\left(t, \tilde{x}_{0}, \tilde{w}(\cdot)\right)$, where $x(t)$ is a solution of $(1)$ and $\tilde{x}(t)$ is a solution of (6).

(2) The system is strongly detectable if and only if the constrained system (6) is globally asymptotically stable.

(3) The system is strongly observable if and only if the constrained system (6) is trivial, i.e. the only solution is $\tilde{x}(t)=0$.

(4) The system is strong* detectable if and only if for the system (5) whenever $\lim _{t \rightarrow \infty} \tilde{y}(t)=0$ it follows that $\lim _{t \rightarrow \infty} \tilde{x}(t)=0$.

In the special case considered in this paper, the indistinguishable dynamics (5) is very simple, since it is decoupled from the plant and because it is a linear time-varying system. This allows a very deep analysis, that is seldom possible.

Recall that a usual characterization of the zeros (or zero dynamics) of a system corresponds to the set of pairs of initial conditions and inputs, such that the output of the system is zero for all the time. This means that the zero dynamics of the indistinguishable dynamics (5) is given by the constrained system (6). Note that the strong indistinguishable trajectories correspond to the trajectories of the zero dynamics, that strong observability is equivalent to the absence of strong indistinguishable trajectories, i.e to the absence of zeros and strong detectability coincides with the asymptotic stability of the zero dynamics.

Lemma 5 gives a dynamical interpretation of the observability/detectability concepts for the specific case considered. It is clear, that this idea can be used for more general systems, although the obtained indistinguishability dynamics systems are, in general, not so simple as here. Compared to the usual observability criteria, that are based on constructing the observability map with the vector fields, this characterization has several advantages. 1) The approach is not local whether in time nor in the state space. 2) It allows to determine detectability, what is usually impossible in the other criteria. 3) The dynamical interpretation is appealing. 4) Several nonlinear tools can be used to make the analysis, as for example the characterization of the zero dynamics in geometric control. 5) Lyapunov functions can be used for the characterization of the properties. 6) It is of very general nature. No special smoothness or structural properties are necessary. 7) For systems with unknown inputs there is no observability test based on the system vector fields in the literature.

\section{DETECTABILITY ANALYSIS FOR UNCERTAIN REACTION SYSTEMS}

The analysis of the error dynamics (4) provides basic information for the design of UIO's. Since 
strong* detectability is a necessary condition for the existence of UIO (see Lemma 4), the objective is to determine conditions on the system, such that when $\tilde{y} \rightarrow 0$ as $t \rightarrow \infty$ it follows that $\tilde{x} \rightarrow 0$ as $t \rightarrow \infty$. Since $D$ is in general a time varying matrix the results for the LTI case of (Hautus, 1983) do not apply. It is clear that asymptotic stability of the zero dynamics (6), i.e. asymptotic minimum phaseness, or equivalently strongly detectability, is a necessary property. Sufficient conditions (in the LTI case also necessary) are:

Proposition 6. Consider the system (5) with constant matrices $C$ and $K$ and a uniformly bounded time-varying matrix $D . \tilde{y} \rightarrow 0$ as $t \rightarrow \infty$ implies $\tilde{x} \rightarrow 0$ as $t \rightarrow \infty$ if

(1) $\operatorname{rank}(C K)=\operatorname{rank}(K)$,

(2) System (5) is exponentially minimum phase, i.e. the system described by the DAE (6) has an exponentially stable equilibrium point.

PROOF. Define a regular output transformation

$$
\bar{y}=\left[\begin{array}{l}
\bar{y}_{1} \\
\bar{y}_{2}
\end{array}\right]=S \tilde{y}=\left[\begin{array}{l}
S_{1} \\
S_{2}
\end{array}\right] \tilde{y}
$$

where $S_{1} \in \mathbb{R}^{q \times m}, S_{2} \in \mathbb{R}^{(m-q) \times m}$ and $S$ is regular. Due to condition (1) $S_{1}, S_{2}$ and $M$, such that $M K=0$, can be so selected, that

$$
T=\left[\begin{array}{l}
T_{1} \\
T_{2} \\
T_{3}
\end{array}\right]=\left[\begin{array}{l}
S_{1} C \\
S_{2} C \\
M
\end{array}\right]
$$

defines a state transformation $z=T \tilde{x}$. Defining $z_{i} \triangleq T_{i} \tilde{x}, i=1,2,3$, system (5) in the new coordinates is given by

$$
\begin{aligned}
\dot{z}_{1} & =\bar{D}_{11} z_{1}+\bar{D}_{12} z_{2}+\bar{D}_{13} z_{3}+w \\
\dot{z}_{2} & =\bar{D}_{21} z_{1}+\bar{D}_{22} z_{2}+\bar{D}_{23} z_{3} \\
\dot{z}_{3} & =\bar{D}_{31} z_{1}+\bar{D}_{32} z_{2}+\bar{D}_{33} z_{3} \\
\bar{y} & =\left[\begin{array}{l}
\bar{y}_{1} \\
\bar{y}_{2}
\end{array}\right]=\bar{C} z=\left[\begin{array}{l}
z_{1} \\
z_{2}
\end{array}\right]
\end{aligned}
$$

where

$$
\begin{aligned}
& \bar{D}=-T D T^{-1}=\left[\begin{array}{ccc}
\bar{D}_{11} & \bar{D}_{12} & \bar{D}_{13} \\
\bar{D}_{21} & \bar{D}_{22} & \bar{D}_{23} \\
\bar{D}_{31} & \bar{D}_{32} & \bar{D}_{33}
\end{array}\right], \\
& \bar{C}=S C T^{-1}=\left[\begin{array}{ccc}
I_{q} & 0 & 0 \\
0 & I_{m-q} & 0
\end{array}\right] .
\end{aligned}
$$

The zero dynamics of the original system in the new coordinates is then

$$
\dot{z}_{3}=\bar{D}_{33} z_{3}, 0=\bar{D}_{13} z_{3}+w, 0=\bar{D}_{23} z_{3}
$$

that by hypothesis is assumed to be exponentially stable. By standard results of input/output stability of LTV systems (Callier and Desoer, 1991) it follows that when $z_{1} \rightarrow 0$ and $z_{2} \rightarrow 0$ then $z_{3} \rightarrow 0$ as $t \rightarrow \infty$ in system (8) and therefore $\tilde{y} \rightarrow 0$ implies $\tilde{x} \rightarrow 0$ for (5).
Remark 7. Note that the first condition implies that $m \geq q$, i.e. the number of measurements has to be larger than the number of unknown inputs.

Remark 8. Exponential minimum phaseness has to be required, since for LTV systems asymptotic stability does not assure input/output stability (see (Rugh, 1993)), that is required in the subsystem $z_{3}$ in (8). To clarify this observation consider the scalar system with bounded coefficients

$$
\begin{aligned}
\dot{x}(t) & =\frac{-2 t}{t^{2}+1} x(t)+u(t), x\left(t_{0}\right)=x_{0}, \\
y(t) & =x(t)
\end{aligned}
$$

This system is not exponentially stable since the transition matrix is given by

$$
\Phi\left(t, t_{0}\right)=\frac{t_{0}^{2}+1}{t^{2}+1} .
$$

But it is uniformly stable and the zero-input response goes to zero for every initial state. However, it is not BIBO stable and a vanishing input does not produce a vanishing output, i.e. $u \rightarrow 0 \nRightarrow$ $y \rightarrow 0$ as $t \rightarrow \infty$. To see this consider the output response for $t_{0}=0$ and $x_{0}=0$

$$
y(t)=\frac{1}{t^{2}+1} \int_{0}^{t}\left(\tau^{2}+1\right) u(\tau) d \tau .
$$

For $u=1$ the output $y(t)=\left(t^{3} / 3+t\right) /\left(t^{2}+1\right)$ is unbounded. For $u(t)=t^{r} /\left(t^{2}+1\right)$, with $1<$ $r<2, u(t) \rightarrow 0$ but $y(t)=t^{(r+1)} /(r+1)\left(t^{2}+1\right)$ is unbounded! And so in general exponential stability has to be imposed. However, exponential minimum phaseness is also not necessary. Consider for example subsystem $z_{3}$ in (8) and that $\bar{D}_{31}=0$ and $\bar{D}_{32}=0$. In this case asymptotic minimum phaseness is sufficient to assure the convergence.

Remark 9. It is interesting to note, that it is possible to have a system that is strong* detectable and strongly observable. This is the case when the only solution of $(9)$ is $z_{3}=0$. However, this is not possible if $m=q$.

\section{OBSERVER DESIGN}

For LTI systems the conditions in the previous proposition are also sufficient for the existence of an UIO. For time-varying systems this seems not to be the case. However, under some mild further assumptions (always satisfied in the LTI case), an UIO can be constructed. For simplicity it will be assumed that the plant has been transformed according to the state and output transformation introduced in the proof of Proposition 6 .

Proposition 10. Consider the reactor system (1) with constant matrices $C$ and $K$, and a uniformly 
bounded time-varying matrix $D$, that satisfies the conditions of Proposition 6 and is represented in new coordinates as

$$
\begin{aligned}
& \dot{\xi}_{1}=\bar{D}_{11} \xi_{1}+\bar{D}_{12} \xi_{2}+\bar{D}_{13} \xi_{3}+w+u_{1} \\
& \dot{\xi}_{2}=\bar{D}_{21} \xi_{1}+\bar{D}_{22} \xi_{2}+\bar{D}_{23} \xi_{3}+u_{2} \\
& \dot{\xi}_{3}=\bar{D}_{31} \xi_{1}+\bar{D}_{32} \xi_{2}+\bar{D}_{33} \xi_{3}+u_{3} \\
& \bar{y}=\left[\begin{array}{l}
\bar{y}_{1} \\
\bar{y}_{2}
\end{array}\right]=\bar{C} \xi=\left[\begin{array}{l}
\xi_{1} \\
\xi_{2}
\end{array}\right] .
\end{aligned}
$$

Consider the subsystem independent of the unknown input

$$
\begin{aligned}
& \dot{\xi}_{2}=\bar{D}_{21} \xi_{1}+\bar{D}_{22} \xi_{2}+\bar{D}_{23} \xi_{3}+u_{2} \\
& \dot{\xi}_{3}=\bar{D}_{31} \xi_{1}+\bar{D}_{32} \xi_{2}+\bar{D}_{33} \xi_{3}+u_{3} \\
& \bar{y}_{2}=[0, I, 0] \xi=\xi_{2},
\end{aligned}
$$

and a reduced order UIO for the plant of the form

$$
\begin{aligned}
& \dot{\hat{\xi}}_{2}=\bar{D}_{21} \bar{y}_{1}+\bar{D}_{22} \hat{\xi}_{2}+\bar{D}_{23} \hat{\xi}_{3}+u_{2}+L_{1}\left(\hat{y}_{2}-y_{2}\right) \\
& \dot{\hat{\xi}_{3}}=\bar{D}_{31} \bar{y}_{1}+\bar{D}_{32} \hat{\xi}_{2}+\bar{D}_{33} \hat{\xi}_{3}+u_{3}+L_{2}\left(\hat{y}_{2}-y_{2}\right)
\end{aligned}
$$

$\hat{y}_{2}=[0, I] \hat{\xi}=\hat{\xi}_{2}$,

(1) If the pair $\left([I, 0],\left[\begin{array}{ll}\bar{D}_{22} & \bar{D}_{23} \\ \bar{D}_{32} & \bar{D}_{33}\end{array}\right]\right)$ is UCO, then (10) is a reduced order UI observer whose convergence dynamics can be arbitrarily assigned.

(2) If the pair $\left([I, 0],\left[\begin{array}{ll}\bar{D}_{22} & \bar{D}_{23} \\ \bar{D}_{32} & \bar{D}_{33}\end{array}\right]\right)$ is uniformly detectable, then (10) is a reduced order UI observer.

It is interesting to note that the results derived in the literature under the name asymptotic observer (Bastin and Dochain, 1990), that are able to estimate the state of a bioreactor without knowledge of the reaction rates, are especial cases of the results in this paper. In fact, our results are a justification from the observability/detectability point of view of the asymptotic observers.

In particular if $D$ is a scalar, i.e. $D=d \mathbb{I}$, then if the measurements are selected such that condition (1) of Proposition 6 is satisfied, then condition (2) is equivalent to the persistency of excitation of the input $d(t)$ (Bastin and Dochain, 1990). In this case it is however impossible to satisfy condition (1) of Proposition 10, an therefore it is not possible to assign the convergence dynamics of the UIO, no matter how the measurements are selected.

\section{CONCLUDING REMARKS}

In this paper a new method has been proposed for the characterization of the observabil- ity/detectability properties of uncertain reaction systems, when the uncertainty is modeled as an arbitrary unknown input. A fairly complete characterization of these properties for the reactor system with unknown reaction rates has been obtained using this method and sufficient conditions for the possibility of constructing robust (asymptotic) observers have been given. These initial results open the possibility to study further other situations in a methodological manner. This will be pursued in future work.

\section{REFERENCES}

Bastin, G. and D. Dochain (1990). On-line Estimation and Adaptive Control of Bioreactors. Vol. 1 of Process Measurement and Control. Elsevier. Amsterdam.

Callier, F.M. and C.A. Desoer (1991). Linear System Theory. Springer-Verlag. New York.

Dochain, D. (2003). State observers for processes with uncertain kinetics. Int. J. Control 76(15), 1483-1492.

Dochain, D. and L. Chen (1992). Local observability and controllability of stirred tank reactors. J. Process Control 2, 139-144.

Dochain, D. and P.A. Vanrolleghem (2001). Dynamical Modelling and Estimation in Wastewater Treatment Processes. IWA Publishing. London, UK.

Dochain, D., M. Perrier and B.E. Ydstie (1992). Asymptotic observers for stirred tank reactors. Chemical Engineering Science 47(15/16), 4167-4177.

Gauthier, J.P. and I. Kupka (2001). Deterministic Observation Theory and Applications. Cambridge University Press. Cambridge, UK.

Hautus, M.L.J. (1983). Strong detectability and observers. Linear Algebra and its Applications 50, 353-368.

Hermann, R. and A.J. Krener (1977). Nonlinear controllability and observability. IEEE Trans. Automatic Control 22, 728-740.

Ibarra-Rojas, S., J. Moreno and G. EspinosaPerez (2004). Global observability analysis of sensorless induction motors. Automatica 40, 1079-1085.

Ponzoni, I., M.C. Sánchez and N.B. Brignole (2004). Direct method for structural observability analysis. Industrial $\&$ Engineering Chemistry Research 43, 577-588.

Rugh, W.J. (1993). Linear System Theory. Prentice Hall. Englewood Cliffs, NJ.

Zeitz, M. (1989). Canonical normal forms for nonlinear systems. In: Nonlinear Control Systems Design - Selected Papers from IFACSymposium (A. Isidori, Ed.). pp. 33-38. Pergamon Press. Oxford. 